\title{
Large-scale Gogny-HFB Calculation for r-Process Nucleosynthesis: Towards a fully-converged microscopic mass table
}

\author{
Alexander Arzhanov ${ }^{* a b}$, Tomás R. Rodríguez ${ }^{a c}$, and Gabriel Martínez-Pinedo ${ }^{a b}$ \\ ${ }^{a}$ Institut für Kernphysik, Technische Universität Darmstadt, \\ D-64289 Darmstadt, Germany. \\ ${ }^{b}$ GSI Helmholtzzentrum für Schwerionenforschung, \\ Planckstraße 1, D-64291 Darmstadt, Germany. \\ ${ }^{c}$ Departamento de Física Teórica, Universidad Autónoma de Madrid, \\ E-28049 Madrid, Spain. \\ E-mail: \\ aarz@theorie.ikp.physik.tu-darmstadt.de,
tomas.rodriguez@uam.es,
gabriel.martinez@physik.tu-darmstadt.de.
}

We employ the approach of Hartree-Fock-Bogolyubov nuclear theory with the effective Gogny interaction in D1S parametrization to perform a large-scale calculation of nuclear masses from proton-drip to neutron-drip line for even-even nuclei. We compare our results to the experimentally known masses listed in 2012 Atomic Mass Evaluation compilation [21, 22], as well as to a previously published AMEDEE database [23, 24, 25]. Owing to lack of convergence in a truncated working basis, we employ and benchmark one of the recently proposed energy correction techniques $[27,28]$ to extrapolate our results to the limit of an infinite model space.

XIII Nuclei in the Cosmos,

7-11 July, 2014

Debrecen, Hungary

\footnotetext{
*Speaker.
} 


\section{Introduction.}

Although it is well-established that almost half of the heavy elements are produced by the rapid neutron-capture process, the quest to identify the actual astrophysical site for this nucleosynthesis scenario is still ongoing [1, 2, 3]. Regardless of the proposed site, all of the currently developing $r$ process models require nuclear physics input for a large number of nuclei that have extreme neutron excess and stretch up to the limits of the nuclear chart. Such nuclei lie far beyond capabilities of experimental facilities in any foreseeable future, and hence performing r-process simulations one has to almost entirely rely on theoretical predictions. Since masses determine thresholds of all nuclear reactions, the calculated final r-process elemental abundances of any astrophysical model are very sensitive [4, 5] to the employed nuclear mass tables, e.g. FRDM [6], WS [7], DZ [8].

Self-consistent mean-field theories based on Hartree-Fock-Bogolyubov (HFB) variational approach with energy density functionals (EDF) were actively developing in the recent decades and have proven successful in systematic study of low energy nuclear structure [9, 10, 11]. In particular, the recent HFB-based mass models are now found to be on a similar accuracy level in describing experimental masses as the more phenomenological approaches [12, 13]. However, besides the known issues of missing beyond-mean-field correlations [13, 14, 15, 24] and the so-called oddeven effects [16, 17], there are also purely numerical problems, as an incomplete convergence of observables in practical computations that can lead to numerical noise in form of artificial jumps in the calculated binding and neutron-separation energies. In what follows, we discuss the issue of insufficient convergence of practical HFB calculations in more detail.

\section{Large-Scale Calculation.}

In current EDF implementations the N-body wavefunctions are usually expanded in a spherical harmonic oscillator ( $\mathrm{SHO}$ ) working basis that is defined by the total number of major oscillator shells $N_{O S}$, and their intrinsic length parameter $b$ [18]. Due to variational nature of HFB approach, the best approximation of the ground state binding energy $E_{H F B}$ is given by [19]

$$
E_{H F B}=\min \left\{E\left(N_{O S}, b\right)\right\},
$$

where $E\left(N_{O S}, b\right)$ is the binding energy (BE) computed in basis with parameters $N_{O S}$ and $b$. However, the binding energies (BEs) should ideally be independent of the chosen basis parameters. Fig. 1 shows the calculated ground-state BEs of ${ }^{16} \mathrm{O}$ for bases $N_{O S}=11, \ldots, 21$ that are plotted against various oscillator lengths $b$. One sees that going from $N_{O S}=11$ to $N_{O S}=13$, or from $N_{O S}=13$ to $N_{O S}=15$ yields noticeably deeper minima of BEs. Yet given a

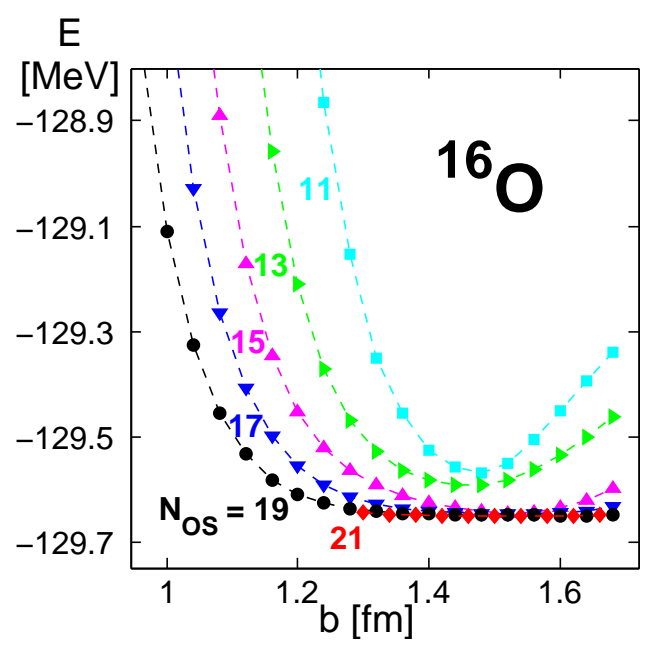

Figure 1: HFB calculations of ground state binding energies of ${ }^{16} \mathrm{O}$ as a function of SHO basis length parameter $b$ for different basis dimensions $N_{O S}=11, \ldots, 21$ (colored labels). Dashed lines to guide the eye. 

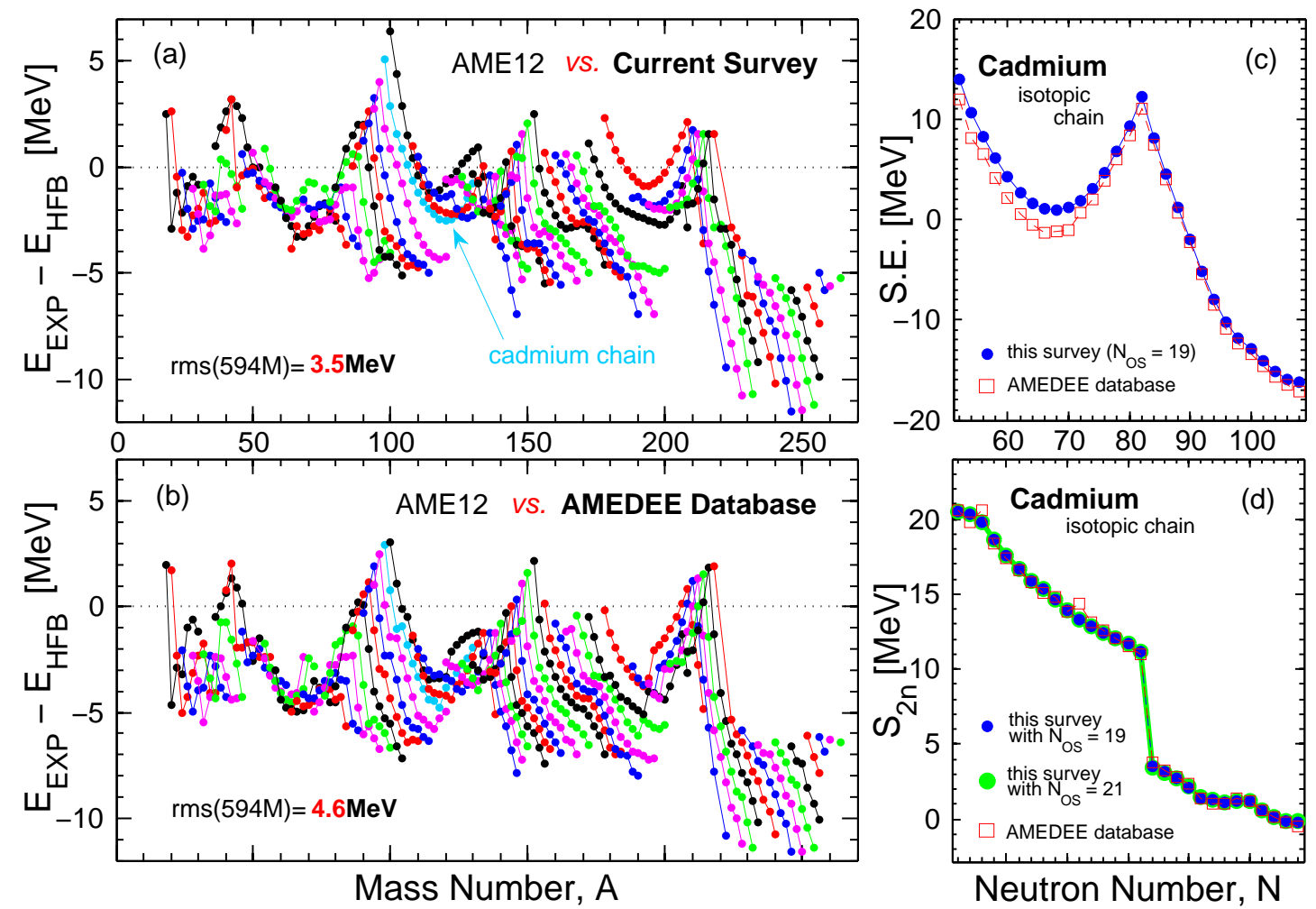

Figure 2: Energy differences between AME12 compilation and theoretical masses according to calculations performed: (a) in this work, and (b) published results in Ref. [25]; (c) comparison of Shell Effects, and (d) two-neutron separation energies of cadmium isotopic chain.

sufficiently large basis, the binding energies are largely insensitive to the parameters of the basis $N_{O S}$ and $b$. We can thus state, that in this case, the results are virtually converged to the true HFBenergy, thereafter to be referred to as $E_{\infty}$. However, due to the limited computational resources and an improper asymptotic behavior of oscillator wave functions at long distances, this ideal is often hard to achieve in practice for heavier and/or neutron-rich isotopes. This is particularly the case for nuclei close to the drip lines, where the BEs are not fully converged in the largest basis used in this survey $\left(N_{O S}=19\right)$.

A global drip-to-drip line calculation of nuclear masses with finite-range Gogny D1S interaction [20] is computationally an intensive and challenging task. In spite of narrowing down the scope of this study to the set of even-even isotopes, the full list of such nuclei $8 \leq Z \leq 134$ includes 2180 entries. Allowing only axial deformations, up to 100 independent calculations had to be carried out for every nucleus in order to ensure a bettermost convergence of the final ground state energy. The results are presented on Fig. 2 (a), where the difference between the experimental binding energies of 2012 Atomic Mass Evaluation (AME12) [21, 22] and current calculations are plotted as a function of mass number A. For comparison, Fig. 2 (b) shows a similar plot, but for the computed masses listed in previously published AMEDEE database [23, 24, 25]. The common oscillating pattern is well-seen for both surveys, with the maxima appearing at the neutron magic numbers $(A \approx 100,150,220)$, where the estimates of the theoretical approach are overbound. Another troublesome trend for D1S parametrization appears in the region of the heaviest nuclei. The 
plotted difference for those neutron-rich isotopes greatly bends downwards, meaning that theoretical results are much underbound in this mass region. Such tendency is due to the fact the the neutron matter equation of state was not taken into account in the fit of the parameters of the Gogny D1S interaction, whereas newer parametrizations, such as D1N [26] and D1M [13], resolve this deficiency. When compared to the previously published databases [23, 24, 25], our results are better converged by an average of $1.47 \mathrm{MeV}$, with the largest improvements in the regions between the neutron-shell closures in mass regions $A \sim 130$ and $A \sim 180$. That is also evident from the Fig. 2 (c) showing a comparison of the residual Shell Effects ${ }^{1}$ for cadmium isotopic chain. The fact of a better convergence is also reflected in the observation that, in general, our calculations yield much smoother mass curves than the AMEDEE results, Fig. 2 (a, b). This, in turn, also produces smoother curves of the two-neutron separation energies ${ }^{2}$ without numerical noise in form of abrupt artificial jumps present in the AMEDEE calculations. This can be seen, for example, in Fig. 2 (d), where the computed cadmium two-neutron separation energies are compared. We can also notice that, despite the still unconverged results for the masses, the extracted two-neutron separations energies are found to be virtually converged, which can be seen from the overlap of $S_{2 n}$ values calculated in $N_{O S}=19$ and $N_{O S}=21$ bases, Fig. 2 (d). As for the root-mean-square (rms) deviations, a comparison of the 594 even-even masses listed in AME12 compilation with this calculation yields an rms error of $3.5 \mathrm{MeV}$, whereas the AMEDEE database has a value of $4.6 \mathrm{MeV}$.

\section{Extrapolation to infinite working basis.}

The evident lack of convergence in practical HFB calculations prompts us to search for a systematic and reliable method to extrapolate the results obtained in a truncated oscillator space to the limit of an infinite basis. In this section we address the extrapolation scheme for groundstate energies first proposed by J. R. Furnstahl, G. Hagen, and T. Papenbrock [27]. A refined extrapolation method (referred to as $L_{2}$-Extrapolation) can be found in Ref. [28]. Despite the apparent success of $L_{2}$-Extrapolation in connection to couple-cluster calculations of ${ }^{6} \mathrm{He}$ and ${ }^{16} \mathrm{O}$ nuclei, the proposed extrapolation scheme has not yet been put to a systematic test in the neutronrich extremes of heavier nuclear systems. In this section we briefly present the extrapolation method on an example of ${ }^{16} \mathrm{O}$ and then discuss its applicability to the cases of heavier isotopic chains.

$L_{2}$-Extrapolation. For a chosen single-particle basis of SHO functions, there are two momentum cutoffs intrinsic to the finite model space itself. One of the cutoffs is associated with the energy of the highest SHO level, or with the maximum momentum a particle in such a basis can reach, called the UV-momentum, $\Lambda_{U V} \equiv \sqrt{2(N+3 / 2)} \cdot \hbar / b$. Preferably one has to choose the basis parameters in such a way that the highest momentum scale $\lambda$ of the employed interaction is smaller than the maximum momentum in the working basis, i.e. $\lambda<\Lambda_{U V}$. The second cutoff corresponds to the maximum radial extent $L$ of the finite oscillator space, which, if possible, should be chosen to be greater than the corresponding radius of the nucleus $R$, so that one can fully encompass the manybody wave function in the coordinate space. A previous set of empirical tests for some simpler

\footnotetext{
${ }^{1}$ Shell Effects are defined as the difference between the binding energy predicted by the liquid-drop model (LDM) and the computed HFB-energy, i.e. S.E. $=E_{L D M}(Z, N)-E_{H F B}(Z, N)$.

${ }^{2}$ two-neutron separation energies $S_{2 n}$ are defined as $S_{2 n}(Z, N)=E_{H F B}(Z, N-2)-E_{H F B}(Z, N)$.
} 

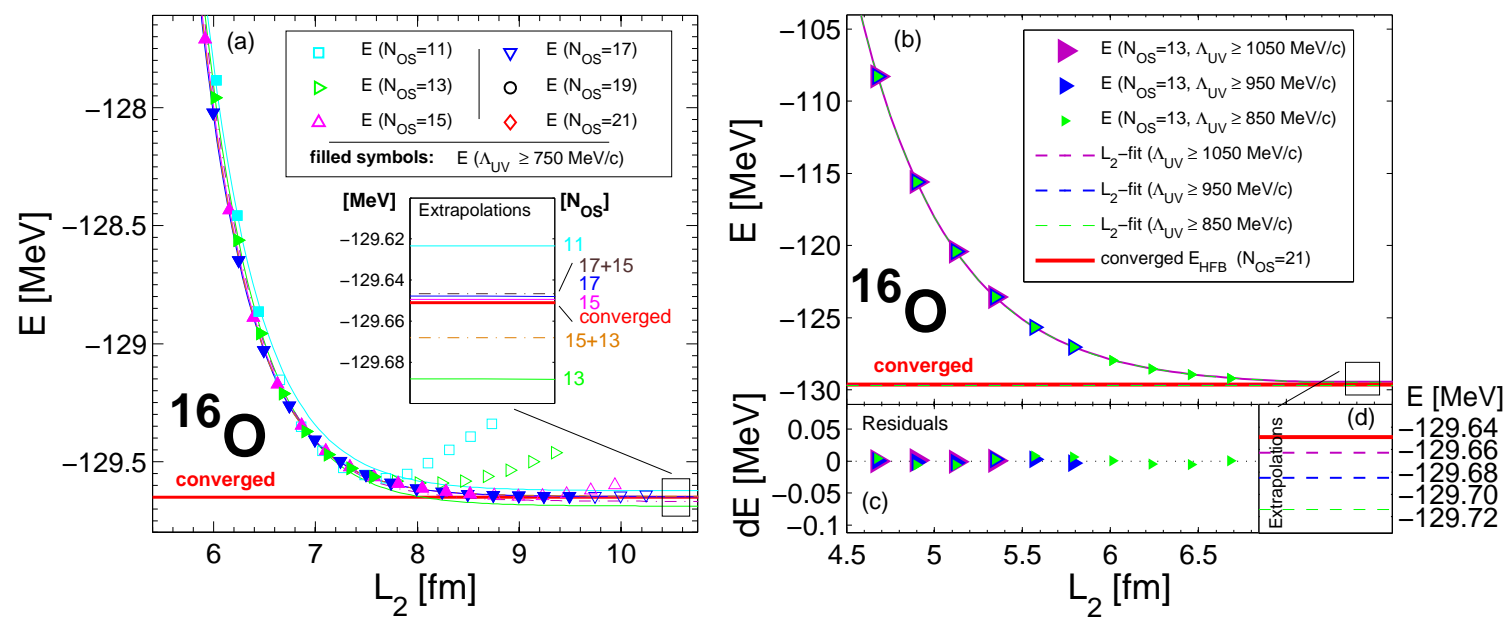

Figure 3: (a) calculated HFB energies in SHO bases with $N_{O S}=11, \ldots, 21$ as function of $L_{2}$. Filled symbols indicate BEs having $\Lambda_{U V}>750 \mathrm{MeV} / \mathrm{c}$, inset shows $E_{\infty}$ values for different extrapolations; (b) BEs calculated in $N_{O S}=13$ basis and the corresponding fits (solid lines); (c) residuals defined as $d E=E\left(L_{2}\right)-E_{L}\left(L_{2}\right)$; (d) the corresponding extrapolations. Red line shows the converged result.

potentials, supported by theoretical motivation [28], revealed an evident preference in setting the box size of the basis to $L=L_{2} \equiv \sqrt{2(N+3 / 2+2)} \cdot b$.

However, in practice, satisfying both conditions of having $\lambda<\Lambda_{U V}$ and $R<L_{2}$ does not guarantee converged results, because the momentum cutoff $\lambda$ is usually not sharp, and the nuclear wave function extends beyond the nuclear radius $R$. Nevertheless, the effective nuclear interactions usually exhibit a Gaussian falloff in momentum space, whereas the nuclear wave function only falls off exponentially in coordinate space [29]. Thus, once $\lambda<\Lambda_{U V}$ holds, the UV convergence in momentum space will be rapid, and the lack of convergence is primarily due to the slower falloff in coordinate space. In the limit of UV-converged calculations, we can implement the following IR-corrections of the form [28]

$$
E_{L}\left(L_{2}\right)=\Delta E_{L}\left(L_{2}\right)+E_{\infty}=B \exp \left(c \cdot L_{2}\right)+E_{\infty},
$$

where energy correction $\Delta E_{L}$ is a function of the effective radial extent $L_{2}$, while $B, c$ and $E_{\infty}$ are the fit parameters.

In order to apply the IR-corrections, we must first separate out the UV-converged results. This is achieved by selecting only those calculations for which UV-cutoff was sufficiently large, e.g. $\Lambda_{U V}>750 \mathrm{MeV} / \mathrm{c}$ [30]. If we now plot the selected HFB energies as a function of the effective radial extent $L_{2}$ (Fig. 3 (a), filled symbols), we find that all of them almost perfectly fall on an exponential curve, consistent with the theoretical predictions for UV-converged results. The observed bend upwards of BEs for larger $L_{2}$ (Fig. 3 (a), hollow symbols) is due to insufficient UVconvergence. Those calculations are excluded from the fit. The curves on Fig. 3 (a) represent the fits to the HFB results from different combinations of basis dimensions, whereas the inset shows the corresponding extrapolated values, $E_{\infty}$.

Of course, a reliable extrapolation should also be independent of the set of UV-converged BEs used in the fit. We verify that it is indeed the case for ${ }^{16} \mathrm{O}$ by fitting to different sets of BEs 


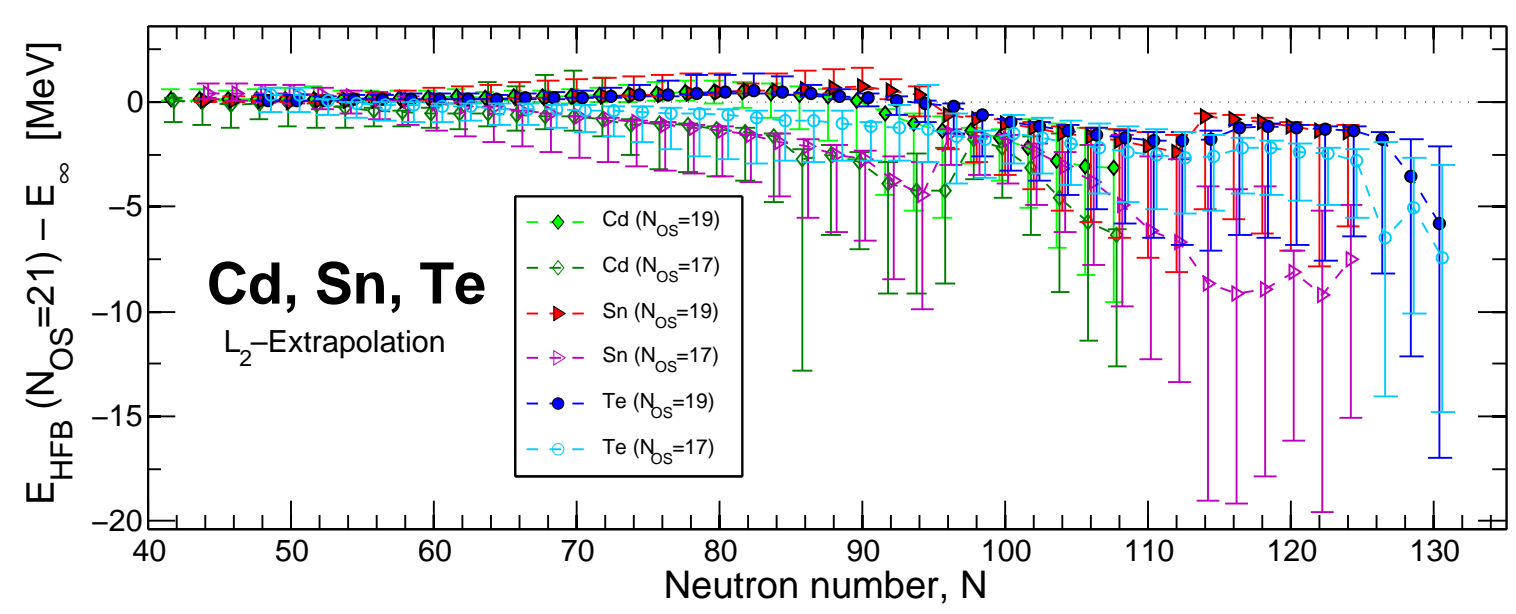

Figure 4: Results of $L_{2}$-Extrapolation for nuclei in cadmium, tin, and tellurium isotopic chains as the function of neutron number. The symbols indicate the difference between $E_{H F B}$ results in $N_{O S}=21$ basis and extrapolated $E_{\infty}$-values for each nuclei obtained from sets of HFB calculations with model spaces truncated at $N_{O S}=17$ and $N_{O S}=19$, separately. The momentum-threshold has been set to $\Lambda_{t h r}=750 \mathrm{MeV} / \mathrm{c}$. The associated errorbars represent the spread in corresponding $E_{\infty}$-values by varying the threshold-parameter in the interval $700 \mathrm{MeV} / \mathrm{c}<\Lambda_{t h r}<900 \mathrm{MeV} / \mathrm{c}$.

calculations, defined by the choice of a threshold value $\Lambda_{t h r}$, which we here choose as $\Lambda_{U V}>$ $\Lambda_{t h r}=850,950,1050 \mathrm{MeV} / \mathrm{c}$. Moreover, in order to imitate a typical situation (common to heavy and loosely bound nuclear systems) of having access to an insufficiently large working basis for convergence, we limit ourselves to a SHO basis with $N_{O S}=13$. The results of this benchmark can be seen on Fig. 3 (b), where fits for different threshold values are provided by the colored dashed lines. All HFB points are found to be on an exponential curve and the quality of exponential convergence pattern is particularly well seen on Fig. 3 (c). All fits yield corresponding $E_{\infty}$ estimates with narrow spread falling very close to the converged energy value, $c f$. Fig. 3 (d), indicating a good stability of the method. Having introduced the tools of $L_{2}$-Extrapolation and benchmarked its performance on the example of ${ }^{16} \mathrm{O}$, we are now in position to systematically test the proposed scheme on a set of heavier nuclei.

Cadmium, tin, and tellurium. Following the discussion in the previous section, we define a ideal extrapolation to posses the following properties: (i) independence of the chosen threshold value $\Lambda_{t h r}$ that define the set of BEs used in the fits according to criteria $\Lambda_{U V}>\Lambda_{t h r}$, as long as the choice ensures UV-convergence; (ii) insensitivity to the basis dimensionality in which the BEs for the fit were obtained (e.g. the $E_{\infty}$-values are independent of whether we pick only the $N_{O S}=17$ calculations, or only $N_{O S}=19$ ones, or a mixture thereof); (iii) finally, given that the fully converged value of $\mathrm{BE}$ is unknown, extrapolations should be able to at least reproduce the bettermost converged $\mathrm{BE}$ calculation at hand, or yield $E_{\infty}$-estimates that are deeper than that.

In what follows, we test the performance of $L_{2}$-Extrapolation at the most relevant parts of nuclear chart (i.e. heavy and neutron-rich nuclei) in accordance to the robustness criteria established above. In particular, we systematically apply the extrapolation scheme to the isotopic chains of cadmium, tin, and tellurium up to the neutron-drip line. Fig. 4 shows the extrapolation results for 
each isotope of these chains for $\Lambda_{U V}>\Lambda_{t h r}=750 \mathrm{MeV} / \mathrm{c}$ obtained from sets of HFB calculations performed in $N_{O S}=17$ basis, and separately in $N_{O S}=19$ basis, see caption. The error bars represent the spread in extrapolated values from varying the momentum-threshold in the following interval: $700 \mathrm{MeV} / \mathrm{c}<\Lambda_{t h r}<900 \mathrm{MeV} / \mathrm{c}$. Although we do not directly associate this spread in $E_{\infty}$-values with an error estimate of the extrapolation procedure, it is, nevertheless, representative of the robustness of the method. Moreover, as a reliable extrapolation should be independent from whether we choose $N_{O S}=17$ or $N_{O S}=19$ calculations for the fit, thus one should rather consider the accumulative spread coming from both sets of data.

As one can see for all the considered isotopic chains, the most robust extrapolations are obtained for isotopes in the direct vicinity to the stability region. However, in this region the extrapolations are least relevant due to larger degree of convergence of HFB calculations in comparison to neutron-rich isotopes. As one moves away from the stable isotopes towards the neutron-drip line, we observe an increasing divergency from the theoretically predicted exponential convergence patterns of the BEs, which, in turn, leads to significant deterioration in performance of the extrapolation. In particular, the discrepancy of extrapolations from $N_{O S}=17$ and $N_{O S}=19$ values (with $\Lambda_{t h r}=750 \mathrm{MeV} / \mathrm{c}$ ) reach easily up to 5-8 MeV. Besides that, varying $\Lambda_{t h r}$ for neutron-rich nuclei has a much greater impact on the estimated $E_{\infty}$ values (spanning energies of $10-15 \mathrm{MeV}$ for $A \sim 115$ ). Finally, we also notice that the IR-corrections can no longer even reproduce the most converged HFB-calculations at hand, let alone yield deeper binding energy than BEs computed in $N_{O S}=21$ basis for all the chains' nuclei in the neutron-rich region.

\section{Summary and Discussion.}

We have performed a large-scale Gogny-D1S calculation of ground state binding energies and shown that a better convergence treatment has a perceptible impact on the nuclear masses. In particular, we have achieved a reduction of the rms deviations with the 594 experimental masses [21, 22] from previously obtained $4.6 \mathrm{MeV}$ [25] to $3.5 \mathrm{MeV}$. Numerical noise due to lack of convergence is removed, resulting in smoother curves of the total binding energy difference along each isotopic chain, as well as the corresponding two-neutron separation energies.

Due to insufficient convergence of nuclear masses we have implemented a recently proposed $L_{2}$-Extrapolation scheme $[27,28]$ and showed its nearly perfect performance for a playground case of ${ }^{16} \mathrm{O}$. However, a systematic benchmark of $L_{2}$-Extrapolation for the whole set of nuclei from cadmium, tin, and tellurium isotopic chain revealed some limitations of the proposed scheme. The evident failure of the $L_{2}$-Extrapolation to provide reliable results for the neutron-rich nuclei of these chains has been supported by the similar findings for magnesium, silicon, sulphur, argon, and calcium chains [30]. Based on our findings here and in Ref. [30], the conclusion that we can draw in regard to the proposed $L_{2}$-Extrapolation, at least in conformity with HFB calculations, is therefore that the investigated method is applicable with some reliability only in the regions of well-bound nuclei of light to medium-mass isotopic chains. These restrictions of the proposed IR-corrections renders these methods to be of limited applications in astrophysical calculations, as they do not provide reliable estimations at required precision level for heavy nuclei in vicinity of the neutrondrip line. Second-order IR-corrections [28] may perform better in these regions of loosely-bound nuclei. A systematic study of such corrections will be presented in a future work [30]. 


\section{References}

[1] Thielemann F.-K. et al., Prog. Part. Nucl. Phys. 66, 346 (2011).

[2] Qian Y.-Z. and Wasserburg G.J., Phys. Rep. 442, 237 (2007).

[3] Qian Y.-Z. and Wasserburg G.J., Astrophys. J. 588, 1099 (2003).

[4] Arcones A. and Martínez-Pinedo G., Phys. Rev. C 83, 045809 (2011).

[5] Brett S. et al., Eur. Phys. J. A 48, 184 (2012).

[6] Möller P. et al., At. Data Nucl. Data Tables 59, 185 (1995).

[7] Wang N., Liu M., and Wu X., Phys. Rev. C 81, 044322 (2010).

[8] Duflo J. and Zuker A.P., Phys. Rev. C 52, R23 (1995).

[9] Bender M., Heenen P.-H., and Reinhard P.-G., Rev. Mod. Phys. 75, 121 (2003).

[10] Pearson J.M. and Goriely S., Nucl. Phys. A 777, 623 (2006).

[11] Lunney D., Pearson J.M., and Thibault C., Rev. Mod. Phys. 75, 1021 (2003).

[12] Goriely S., Chamel N., and Pearson J.M., Phys. Rev. C 88, 024308 (2013).

[13] Goriely S. et al., Phys. Rev. Lett. 102, 242501 (2009).

[14] Rodríguez T.R., Arzhanov A., and Martínez-Pinedo G., arXiv:1407.7699 (2014).

[15] Bender M., Bertsch G.F., and Heenen P.-H., Phys. Rev. C 73, 034322 (2006), and Phys. Rev. C 78, 054312 (2008).

[16] Duguet T. et al., Phys. Rev. C 65, 014310 (2001).

[17] Bertsch G.F. et al., Phys. Rev. C 79, 034306 (2009).

[18] Dobaczewski J. and Dudek J., Comput. Phys. Commun. 102, 183 (1997).

[19] Ring P. and Schuck P., The Nuclear Many Body Problem (Springer-Verlag, Heidelberg, 1980).

[20] Berger J.F., Girod M., and Gogny D., Nucl. Phys A 428, 23c (1984), and Comput. Phys. Commun. 63, 365 (1991).

[21] Audi G. et al., Chinese Phys. C 36, 1287-1602 (2012).

[22] Wang M. et al., Chinese Phys. C 36, 1603-2014 (2012).

[23] Hilaire S. and Girod M., Eur. Phys. J. A 33, 237 (2007).

[24] Delaroche J.-P. et al., Phys. Rev. C 81, 014303 (2010).

[25] AMEDEE database available online: http://www-phynu.cea.fr/HFB-5DCH-table.htm, and as supplementary material in EPAPS repository: http://link.aps.org/supplemental/10.1103/PhysRevC.81.014303

[26] Chappert F., Girod M., and Hilaire S., Phys. Lett. B 668, 420 (2008).

[27] Furnstahl R.J., Hagen G., and Papenbrock T., Phys. Rev. C 86, 031301 (2012).

[28] More S.N. et al., Phys. Rev. C 87, 044326 (2013).

[29] Bogner S.K. et al., Nucl. Phys. A 801, 21 (2008).

[30] Arzhanov A., Rodríguez T.R., and Martínez-Pinedo G. (in preparation). 